\title{
65. NOVEDADES COROLÓGICAS PARA LA PROVINCIA DE HUELVA
}

\section{E. SÁNCHEZ GULLÓN}

New floristic records for Huelva province.

Palabras clave. Corología, Paraje Natural Marismas del Odiel, Litoral, Huelva, Andalucía Occidental, España.

Key words. Corology, P. N. Marismas del Odiel, Litoral, Huelva, Western Andalusia, Spain.

Este trabajo reúne veintisiete nuevas citas corológicas para la provincia de Huelva resultado de un estudio florístico que pretende reanudar los trabajos botánicos iniciados por B. Cabezudo (1974-1985). Se ha herborizado en toda la provincia centrándonos, principalmente, en el Paraje Natural Marismas del Odiel y litoral onubense, comarca natural caracterizada por un predominio de sistemas dunares movilizados por una gran corriente sedimentaria costera, acantilados de areniscas con arenas pliocuaternarias, lagunas higroturbosas, y sobre todo, marismas mareales o continentalizadas asociadas a los estuarios de los ríos Guadiana, Tinto, Odiel y Guadalquivir.

Cada taxón se acompaña con la localidad de su herborización con U.T.M., ecología, fecha de recolección, recolectores, y número del pliego testigo que se conserva en el Herbario del Departamento de Biología Vegetal y Ecología de la Universidad de Sevilla (SEV).

\section{Pilularia minuta Durieu}

Gravera de Puntales, Paraje Natural Marismas del Odiel (Gibraleón), 29SPB7725, en bordes de gravera temporalmente encharcada, 12-VI-1997, E. Sánchez Gullón \& P. García Murillo, SEV 156769.

Este pteridófito endémico de la Región Mediterránea Occidental propio del Sector Iberoeumediterráneo, se ha citado para Portugal en el Algarve occidental en las proximidades de Samoqueiro, al norte de Vila do Bispo (Amaral Franco \& Rocha Afonso, 1982) y Menorca (Salvo
Tierra, 1990), y no recogido para Huelva por Cabezudo (1978) y en Flora Vascular de Andalucía Occidental (Valdés et al., 1987).

Como ya observó Paiva (1986), es más frecuente de lo que parece, debido a que pasa totalmente desapercibido por lo reducido de su tamaño, que es confundible con hojas jóvenes de juncáceas o poáceas, de las que se diferencia por su prefoliación circinada. Confirmamos su presencia en el Litoral onubense.

\section{Lavatera triloba L. subsp. triloba}

Dehesa del Estero, Moguer, 29SPB9121, en suelo húmedo temporalmente encharcado algo salino, 15-IX-1997, E. Sánchez Gullón, SEV 156741.

Esta malvácea del W de la Región Mediterránea, está citada para el C y SE de España, S de Portugal y $\mathrm{N}$ de Africa (Fernández, 1993). Con nuestra localización confirmamos su presencia en el Litoral onubense.

\section{Tetragonia tetragonoides (Pallas) O. Kuntze}

Laguna Marítima, Paraje Natural Marismas del Odiel (Huelva), 29SPB8316, naturalizado en gancho arenoso sobre marisma mareal, 2-VI-1998, E. Sánchez Gullón \& Juanma Rodriguez Vela, SEV 156770. Isla Canela, Ayamonte, 29SPB4416; en duna de playa, 10-VI-1998, E. Sánchez Gullón, SEV 156742.

Esta aizoácea originaria de Nueva Zelanda es adventicia en Mallorca, Guipúzcoa, Extremadura portuguesa y Algarve (Gonçalves, 1990). Con nuestra cita confirmamos su presencia para Andalucía Occidental, donde está en clara expansión en dunas y arenales costeros. 
Capparis spinosa L. subsp. spinosa var. canescens Coss.

Niebla, 29SQB0537, rupícola sobre molino del río Tinto en sustrato calizo, 15-IV-1997, E. Sánchez Gullón \& S. Silvestre, SEV 156743.

La alcaparrera es un taxón propio de la Región Mediterránea citado en la Península Ibérica principalmente en el S y E ( Marcos Samaniego \& Paiva, 1993). En Huelva se ha localizado en zonas próximas a antiguos asentamientos árabes, donde posiblemente se haya naturalizado por su cultivo. Completamos el área conocida de esta especie para Huelva.

Sinapis alba L. subsp. mairei (H. Lindb.) Maire

Acantilado del Parador Nacional, Ayamonte, 29SPB4121, en bordes de cultivos, 11-V-1998, E. Sánchez Gullón, SEV 156744.

Esta crucífera arvense propia de Europa, $\mathrm{N}$ de África y Región Irano-turánica, no citada con anterioridad para Huelva (Gómez Campos, 1993). Con nuestra localización ampliamos completando el área conocida de esta especie para el Litoral onubense.

\section{Lotus edulis L.}

Marisma del Estero de la Nao, Ayamonte, 29SPB4121, en bordes de marisma sobre suelos calizos, 11-V-1998, E. Sánchez Gullón, SEV 156745 .

Esta fabácea de la Región Mediterránea, se ha citado en el S de Portugal, E y S de la Península Ibérica y Baleares (Pascual, 1978). En Andalucía Occidental es poco frecuente (Ruiz de Clavijo, 1987), coloniza terrenos arenosos, pedregosos, o colinas calcáreas, próximas del litoral. Su presencia en la provincia de Huelva completa su distribución, constituyendo un nexo de unión entre las poblaciones algárvicas y gaditanas.

\section{Ononis dentata Solander ex Lowe}

La Cascajera, Paraje Natural Marismas del Odiel, Huelva, 29SPB8217, en spit arenoso marítimo algo calizo por el aporte de conchenas debajo de sabinar-acebuchal termomediterráneo, 2-VI-1997, E. Sánchez Gullón, SEV 156746.

Esta fabácea propia de suelos arenosos de origen marítimo y sistemas dunares del E y S de España, Portugal, Sicilia y Región Macaronésica, e indicada para el litoral gaditano (Devesa, 1987). No se había citado con anterioridad para Huelva. Con- firmamos su presencia en esta provincia, únde es muy rara en la única localidad conocida.

\section{Glycyrrhiza glabra L.}

Laguna del Prado, La Redondela, 29SPB5321, en márgenes de camino, 21-VIII-1998, E. Sánchez Gullón, SEV (156747). Camino del Garranchal, Cartaya, 29SPB6526, en bordes de marisma, 15-IX1998, E. Sánchez Gullón \& Javier Macías, SEV 156748.

El regaliz es posiblemente originario del E de la Región Mediterránea (Domínguez, 1987), ampliamente cultivado en diversas partes del mundo y adventicio asociado a campos cultivados y lugares arenosos fértiles de vega. Con nuestra cita confirmamos su presencia en la provincia de Huelva donde es muy abundante y conocido.

\section{Astragalus epiglottis L. subsp. epiglottis}

Acantilado del Parador Nacional, Ayamonte, 29SPB4121; en comunidad terofítica sobre suelos calizos, 11-V-1998, E. Sánchez Gullón, SEV 156749 .

Esta fabácea de la Región Mediterránea es conocida en la Península Ibérica en el C, E y S (Pascual, 1978). En Andalucía Occidental está citada en la Campiña Baja, Campiña Alta y Subbética (Domínguez, 1987). Confirmamos su presencia en Huelva ampliando su corología al Litoral onubense donde se localiza en pastizales oligótrofos calizos.

\section{Astragalus boeticus L.}

El Rompido, Cartaya, 29SPB7620; en bordes de carretera, 25-V-1998, E. Sánchez Gullón, SEV 156750. Saltés, Paraje Natural Marismas del Odiel (Huelva), 29SPB 8020, en márgenes de excavación arqueológica, 25-V-1997, E. Sánchez Gullón, SEV 156753.

Esta fabácea de suelos arenosos costeros y cultivos de la Región Mediterránea, se ha citado en Aragón, Cataluña, S de España y Baleares (Pascual, 1978). En Andalucía Occidental se conoce en el Litoral gaditano y Campiña Baja (Domínguez, 1987). Confirmamos con nuestra cita su presencia para el Litoral onubense, donde es muy frecuente comportándose como subnitrófila.

Cullen americanum (L.) Rydb.

La Ribera, Huelva, 29SPB8333, en suelos arcillosos básicos, 20-VI-1997, E. Sánchez Gullón, 


\section{SEV 156752.}

Esta fabácea de la Región Mediterránea del S de Italia, Sicilia y SW de la Península Ibérica. Se conoce en Extremadura (Devesa, 1995) y Andalucía Occidental, donde se cita como rara en Sevilla y Cádiz (Domínguez, 1987).

Ampliamos el área conocida de esta especie a la provincia de Huelva.

\section{Viscum cruciatum Sieber ex Boiss}

Las Canarias, Bonares, 29SQB0834, 20-III1998, E. Sánchez Gullón \& I. Barroso, SEV 156756.

Este taxón parásito de diversas dicotiledóneas propio del SW de la Península Ibérica, $\mathrm{N}$ de África, Israel, $\mathrm{C}$ y $\mathrm{S}$ de Asia. Se conoce en Andalucía en las provincias de $\mathrm{Ca}, \mathrm{Co}$, Gr y $\mathrm{Ma}$, y dado por extinguido en Huelva (Catalán \& Aparicio, 1997). Se ha vuelto a localizar como hospedante de Prunus dulcis (Miller) D. A. Webb., confirmando su presencia actual en la provincia.

\section{Chamaesyce maculata (L.) Small}

Calatilla, Paraje Natural Marismas del Odiel (Huelva), 29SPB8025, ruderal en márgenes de acera, 20-VI-1997, E. Sánchez Gullón, SEV 156754.

Esta euforbiácea neófita de Norteamérica está naturalizada en la Península Ibérica principalmente en la mitad septentrional y Baleares (Benedi, 1997). Se ha citado en la Vega (Valdés, 1987), Cádiz (I. Sánchez García, 1997) y Extremadura (Ruiz, 1995). Con nuestra cita confirmamos su presencia en el Litoral onubense, donde es muy abundante como ruderal en suelos muy nitrificados.

\section{Ammannia robusta Heer \& Regel}

Embalse del Piedras, Villanueva de los Castillejos, 29SPB5642, naturalizado en orillas del embalse, 23-IX-1998, E. Sánchez Gullón, SEV 156755.

Neófito originario de Norteamérica, Centroamérica y Brazil, hasta la fecha adventicio en Badajoz (Tormo, 1995), Navarra, E de España y Sevilla (Velayos, 1997). Ampliamos el área conocida de esta especie a la comarca natural del Andévalo.

\section{Tordilium maximum $\mathrm{L}$.}

Las Cañadas, Galaroza, 29SQC0201, en bordes de camino debajo de alcornocal-castañar. 25VIII-1995, E. Sánchez Gullón, SEV 156757. Pico del San Cristóbal, Almonaster, 29SPB9595, en sotobosque de castaños, 2-XII-1998, E. Sánchez Gullón, SEV 156758.

Apiácea del C y S de Europa, SW de Asia, e introducida en el W de Europa. En Andalucía Occidental se ha citado como ruderal en los Pedroches, Sierra Norte, Vega y Grazalema (García Martín, 1987). Ampliamos el área conocida de esta especie a la Sierra de Aracena, donde se comporta como ruderal y arvense.

\section{Ammoides pusilla (Brot.) Breistr.}

Niebla (Huelva), 29SQB0539, en protosuelo calizo, 20-IV-1997, E. Sánchez Gullón, SEV 156759.

Este taxón de la Región Mediterránea de distribución muy amplia en Andalucía Occidental (García Martín, 1987), no estaba citado para la provincia. Con nuestra localización completamos su distribución andaluza.

Thapsia minor Hoffmanss. \& Link

Campo Común de Abajo, Cartaya, 29SPB703275, en paleocauce pliocénico sobre limos arenosos con costras y nódulos ferruginosos. 15VI-1998, E. Sánchez Gullón \& Santiago Silvestre, SEV 156767.

Primera cita andaluza de esta apiáceae subordinada hasta hace poco a rango de variedad de Thapsia villosa L. (Coutinho \& Pereira, 1913). La corología conocida de esta especie es del C y W de la Península Ibérica, citándose en Madrid, Cáceres, Salamanca, Zamora, Orense y Portugal (E. Bayer \& Ginés López, 1996). Completamos el área conocida de este taxón a Andalucía Occidental.

Kundmannia sicula (L.) DC.

Acantilado del Parador Nacional, Ayamonte, 29SPB4121, en ladera sobre sustrato calizo, 11-V1998, E. Sánchez Gullón, SEV 156751.

Apiácea de la Región Mediterránea citada en Andalucía Occidental en el Litoral gaditano, Campiña Baja, Campiña Alta Gaditana, Grazalema y Algeciras, donde forman extensas poblaciones en suelos margosos (García Martín, 1987) y conocida también en Málaga (Cabezudo et al., 1992). Ampliamos el área conocida de esta especie al Litoral onubense.

\section{Physalis alkekengi $\mathrm{L}$.}

Menajo, Cartaya, 29SPB6533, naturalizada en márgenes de cultivos de secano, 24-VIII-1995, E. 


\section{Sánchez Gullón, SEV 156761.}

Taxón originario del C y S de Europa, W y C de Asia, cultivado y naturalizado en diversas partes del mundo. En Andalucía Occidental se ha citado como raro en la Vega y Campiña Baja (Valdés, 1987). Con nuestra cita ampliamos el área conocida de esta especie a la Campiña onubense.

\section{Veronica cymbalaria Bodard}

Arroyomolinos de León, 29SQC2612, en muros de piedras junto a un camino y huertas, $5-\mathrm{V}$ 1997, E. Sánchez Gullón, SEV 156762.

Esta escrofulariácea rupícola de la Región Mediterránea se ha citado como abundante en Andalucía Occidental (Valdés, 1987), faltando en la provincia de Huelva. Con nuestra cita completamos su corología localizándola en la Sierra de Aracena.

\section{Dichondra micrantha Urban}

El Portil, Cartaya, 29SPB7320, localizado como adventicio en praderas de céspedes y jardines, 15-VII-1998, E. Sánchez Gullón \& S. Silvestre, SEV 137864.

Neófito antillano cultivado como cespitoso y naturalizado en céspedes y zonas ajardinadas del W de Europa (Lawalreé, 1972), Murcia (Ríos Ruiz \& Alcaraz Ariza, 1996) y Región Macaronésica (Hansen \& Sunding, 1985). Con nuestra cita ampliamos el área conocida de esta especie en el sur de la Península Ibérica.

\section{Bidens frondosa $\mathrm{L}$.}

Pto. de la Laja, El Granado, 29SPB3354, en los márgenes del Guadiana, 20-VIII-1998, E. Sánchez Gullón, SEV 156760.

Neófito americano naturalizado en el W, S y C de Europa (Tutin, 1976) y Región Macaronésica (Hansen \& Sunding, 1985). En la Península Ibérica se ha citado adventicio en Extremadura (Ortega, 1995), y con nuestra localización ampliamos el área conocida de este taxón en Andalucía Occidental al Andévalo.

\section{Erigeron karvinskianus DC.}

Tharsis, Alosno, 29SPB6662, introducido como ruderal en aceras del casco urbano, 20-VIII1998, E. Sánchez Gullón, SEV 156763.

Neófito mejicano cultivado como ornamental y naturalizado en muros y rocas del S y W de Europa (Hallyday, 1976), Región Macaronésica (Hansen \&
Sunding, 1985). En la Península Ibérica se conoce en Extremadura (Devesa, 1995) y Cádiz (I. Sánchez García, 1997). Ampliamos el área conocida de esta especie a la comarca natural del Andévalo.

\section{Galinsoga parviflora Cav.}

Huelva, en la carretera de salida hacia La Ribera a $1 \mathrm{~km}, 29 \mathrm{SPB} 8329$, ruderal en cuneta encharcada, 20-XI-1998, E. Sánche Gullón, SEV 156764 .

Asterácea suramericana adventicia en el W de la Región Mediterránea (Tutin, 1976) y Región Macaronésica (Hansen \& Sunding, 1985). En la Península Ibérica se ha citado en Extremadura (Devesa, 1995). Ampliamos la corología de este taxón a la provincia de Huelva.

\section{Centaurea cordubensis Font Quer}

La Redondela, 29SPB5322, ruderal en suelos ácidos dentro de un pinar de Pinus pinea L., 20VIII-1997, E. Sánchez Gullón, SEV 156765.

Asterácea endémica del SW peninsular (Talavera, 1987) no citada anteriormente para la provincia. Ampliamos su distribución conocida al Litoral onubense donde se comporta como ruderal.

\section{Eragrostis pilosa (L.) Beauv}

Higuera de la Sierra, 29SQB2691, en cultivos de regadío, 28-IX-1998, E. Sánchez Gullón, SEV 156768 .

Este taxón originario del C y S de Europa, S, W, C y E de Asia, adventicio en el S de África, América y Australia, en cultivos de regadío, cauces secos y arroyos, fue citado por Sagredo para Almería (1987), no apareciendo en la obra Flora Vascular de Andalucía Occidental (Valdés et al., 1987), y conocido en Extremadura (Devesa, 1991; 1995). Con nuestra localización confirmamos su presencia y distribución en la Sierra de Aracena.

\section{Scilla ramburei Boiss.}

Las Madres, Moguer, 29SPB9215, en suelos arenosos perimetral a turbera, 15-V-1997, E. Sánchez Gullón, SEV 156766.

Taxón propio de suelos arenosos del S y W de la Península Ibérica y NW de África. En Andalucía Occidental se conoce del Litoral gaditano y Algeciras (Pastor, 1987). Con nuestra cita completamos su distribución a todo el litoral atlántico andaluz. 


\section{BIBLIOGRAFÍA}

AMARAL FRANCO \& ROCHA AFONSO -1982Distribuçao de pteridófitos e gimnospérmicas em Portugal. Serviço Nacional de Parques, Reservas e Patrimonio Paisagístico. Lisboa.

BAYER, E. y G. LÓPEZ GONZÁLEZ -1996- Una especie olvidada de Thapsia L. (Umbelliferae): T. minor Hoffmanns. \& Link. Anales Jard. Bot. Madrid 54: 265-272.

BENEDI, C. -1997- Chamaesyce, in CASTROVIEJO et al. (eds.) Flora Ibérica 8: 289-291. Real Jardín Botánico de Madrid. C.S.I.C.

CABEZUDO, B. -1974- Nota corológica sobre la flora de Huelva. Lagascalia 4(2): 281-284.

CABEZUDO, B. -1975- Nota corológica sobre la flora de Huelva. II. Lagascalia 5(1): 77-83.

CABEZUDO, B. -1976- Nota corológica sobre la flora de Huelva. III. Lagascalia 6(2): 256.

CABEZUDO, B. -1978- Nota corológica sobre la flora de Huelva. IV. Lagascalia 7(2): 173-178.

CABEZUDO, B. - 1978- Pterodofitas de la provincia de Huelva. Lagascalia 8(1): 3-12.

CABEZUDO, B. -1979- Plantas de la Reserva Biológica de Doñana (Huelva). II. Lagascalia 8(2): 167-181

CABEZUDO, B. y A. E. SALVO -1987- Marsileaceae, in VALDÉS et al. (eds.) Flora Vascular de Andalucía Occidental I:57. Ed. Ketres. Barcelona.

CABEZUDO, B., J. A. ARENAS POSADA, F. GARCÍA MARTÍN y J. M. NIETO CALDERA -1992- Catálogo de las umbelíferas (Apiaceae) malacitanas. Acta Bot. Malacitana 17: 145-166.

CATALÁN, P. y A. APARICIO -1997- Viscum, in CASTROVIEJO et al. (eds.) Flora Ibérica 8:164. Real Jardín Botánico de Madrid. C.S.I.C. COUTINHO, A. \& X. PEREIRA -1913- A Flora de Portugal. Paris, Lisboa, Río de Janeiro, S. Paulo \& Bello Horizonte.

DEVESA, J. A. -1987- Ononis, in VALDÉS et al. (eds.) Flora Vascular de Andalucía Occidental 2: 149. Ed. Ketres. Barcelona

DEVESA, J. A. -1991-Las gramíneas de Extremadura. Univ. de Extremadura.

DEVESA, J. A. -1995- Cullen, Galinsoga, Eragrostis, in DEVESA (ed.) Vegetación y Flora de Extremadura: 398, 528, 618. Universitas Editorial. Badajoz.

DOMÍNGUEZ, E. -1987- Glycyrrhiza, Astragalus, Psoralea, in VALDÉS et al. (eds.) Flora Vascular de Andalucía Occidental 2: 180, 182183, 187, 189. Ed. Ketres. Barcelona.
FERNÁNDEZ, R. B. -1993- Lavatera, in CASTROVIEJO et al. (eds.) Flora Ibérica III: 238-240. Real Jard. Bot. de Madrid. C.S.I.C.

GALIANO, E. F. y B. CABEZUDO - 1976- Plantas de la Reserva Biológica de Doñana (Huelva). Lagascalia 6(1): 117-176.

GARCÍA MARTÍN, F. -1987- Kundmannia, Ammoides, Tordilium, in VALDÉS et al. (eds.) Flora Vascular de Andalucía Occidental 2: 306, 318, 323. Ed. Ketres. Barcelona.

GÓMEZ CAMPOS, C. -1993-Sinapis, in CASTROVIEJO et al. (eds.) IV: 386-388. Real Jard. Bot. de Madrid. C.S.I.C.

GONÇALVES, M. L. -1990- Tetragonia, in CASTROVIEJO et al. (eds.) Flora Ibérica II: 76-78. Real Jard. Bot. de Madrid. C.S.I.C.

HALLYDAY, G. -1976- Erigeron, in TUTIN et al. (eds.) Flora Europaea. 4: 116-120. Cambridge.

HANSEN, A. \& P. SUNDING -1985- Flora of Macaronesia. Checklist of vascular plants. Sommerfeltia I.

LAWALREÉ, A. -1972- Dichondra, in TUTIN et al. (eds.) Flora Europaea 3: 78. Cambridge.

MARCOS SAMANIEGO y J. PAIVA -1993- Capparis, in CASTROVIEJO et al. (eds.) Flora Ibérica III:518-521. Real Jard. Bot. de Madrid. C.S.I.C.

ORTEGA, A. -1995- Bidens, in DEVESA (ed.) Vegetación y Flora de Extremadura: 527. Universitas Editorial. Badajoz.

PAIVA, J. - 1986- Pilularia, in CASTROVIEJO et al. (eds.) Flora Ibérica I: 71. Real Jard. Bot. de Madrid. C.S.I.C.

PASCUAL, H. -1978- Leguminosas de la Península Ibérica y Baleares. I.N.I.A. Ministerio de Agricultura.

PASTOR, J. - 1987- Scilla, in VALDÉS et al. (eds.) Flora Vascular de Andalucía Occidental 3: 441. Ed. Ketres. Barcelona.

RíOS RUIZ, S. y F. J. ALCARAZ ARIZA -1996Flora de las riberas y zonas húmedas de la cuenca del río Segura. Universidad de Murcia.

RIVERA, J y B. CABEZUDO - 1985- Aportaciones al conocimiento florístico de la Sierra de Aracena (Huelva, España). Acta Bot. Malacitana 10:61-78.

RUBIO, J. C. -1985- Ecología de las Marismas del Odiel. (Tesis Doctoral). Universidad de Sevilla. RUIZ DE CLAVIJO, E. -1987- Lotus, in VALDÉS et al. (eds.) Flora Vascular de Andalucía Occidental 2: 76. Ed. Ketres. Barcelona.

RUIZ, T. -1995- Euphorbia, in DEVESA (ed.) Vegetación y Flora de Extremadura: 408. Universitas Editorial. Badajoz. 
SAGREDO, R. -1987- Flora de Almería. IEA.

SALVO TIERRA, A. E. -1990- Guía de los helechos de la Península Ibérica y Baleares. Ed. Pirámide. Madrid.

SÁNCHEZ GARCÍA, I. -1997- Fragmenta Chorologica Occidentalia $N^{\circ} 5927$ y N 5929 Anales Jard. Bot. Madrid 55(1):152.

SÁNCHEZ GULLÓN \& J. A. ORTEGA EXPÓSITO -1998- Nuevas áreas para la flora de Andalucía Occidental $n^{\circ}$ 55. Lagascalia 20(2): 311-315.

SÁNCHEZ GULLÓN, E. \& J. A. ORTEGA EXPÓSITO -1997- Fragmenta Chorologica Occidentalia $\mathrm{N}^{\circ}$ 6230-6235. Anales Jard. Bot. Madrid 55(2):451.

TALAVERA, S. -1987- Centaurea, in VALDÉS et al. (eds.) Flora Vascular de Andalucía Occidental 3: 150. Ed. Ketres. Barcelona.

TORMO, R. -1995- Ammannia, in DEVESA (ed.) Vegetación y Flora de Extremadura: 399. Universitas Editorial. Badajoz.
TUTIN, T. G. -1976- Bidens, Galinsoga, in TUTIN et al. (eds.) Flora Europaea 4:139-140, 143 144. Cambridge.

VALDÉS, B. -1987-Euphorbia, Physalis, Veronica, in VALDÉS et al. (eds.) Flora Vascular de Andalucía Occidental 2: 223, 357, 541. Ed. Ketres. Barcelona.

VELAYOS, M. -1997- Ammannia, in CASTROVIEJO et al. (eds.) Flora Ibérica 8: 27.Real Jard. Bot. de Madrid. C.S.I.C.

Aceptado para su publicación en Abril de 1999

Dirección de los autores. Paraje Natural Marismas del Odiel. Ctra. del Dique Juan Carlos I. Apdo. 720. 21071 - Huelva.

\section{NOTAS SOBRE LAVEGETACIÓN DE ANDALUCÍA. I.}

\section{Baltasar CABEZUDO y Andrés V. PÉREZ LATORRE}

Short notes about Andalusian vegetation. I.

Palabras clave. Vegetación, sintaxonomía, corología, Andalucía, España.

Key words. Vegetation, syntaxonomy, chorology, Andalusia, Spain.

\section{1.- Abieto pinsapo-Juniperetum sabinae Pérez}

Latorre y Cabezudo in Pérez Latorre, P. Navas, D. Navas, Gil y Cabezudo 1998 (8 inventarios) (Acta Bot. Malacitana 23:155).

Syn.: Rhamno infectoriae-Juniperetum sabinae Díez Garretas y Asensi in Díez Garretas, Fernández González y Asensi 1998 (1999) (1 inventario) (Itinera Geobotanica 11:346)
Astralago nevadensis-Bupleuretum spinosi Pérez Latorre y Cabezudo in Pérez Latorre, P. Navas, D. Navas, Gil y Cabezudo 1998 (10 inventarios) (Acta Bot. Malacitana 23:156).

Syn.: Astragalo andresmolinae-Erinacetum anthyllidis Díez Garretas y Asensi in Díez Garretas, Fernández González y Asensi 1998 (1999) (1 inventario) (Itinera Geobotanica 11:345). 